\title{
Dietary Patterns and Their Association with Blood Pressure Control among Hypertensive Patients in Gaza Strip, Palestine
}

\author{
Abdel Hamid El Bilbeisi", Saeed Hosseini, Kurosh Djafarian \\ Department of Clinical Nutrition, School of Nutritional Sciences and Dietetics, Tehran University of Medical Sciences, International Campus \\ (TUMS- IC), Tehran, Iran \\ Email address: \\ Abed_az@hotmail.com (A. H. El. Bilbeisi), saeedhmdphd@hotmail.com (S. Hosseini), kdjafarian@tums.ac.ir (K. Djafarian) \\ ${ }^{*}$ Corresponding author
}

\section{To cite this article:}

Abdel Hamid El Bilbeisi, Saeed Hosseini, Kurosh Djafarian. Dietary Patterns and Their Association with Blood Pressure Control among Hypertensive Patients in Gaza Strip, Palestine. Journal of Family Medicine and Health Care. Vol. 4, No. 2, 2018, pp. 5-12. doi: $10.11648 /$ j.jfmhc.20180402.11

Received: August 22, 2018; Accepted: September 3, 2018; Published: October 8, 2018

\begin{abstract}
Background: Silent killer, or hypertension, rarely has noticeable symptoms. But if untreated, it increases the risk of serious problems such as heart attacks and strokes. Globally, the World Health Organization estimates that, the overall prevalence of hypertension was around $40 \%$ in adults. However, the role of diet in the origin of hypertension is not understood well. Therefore, the purpose of the current study was to identify major dietary patterns among hypertensive patients and its association with blood pressure control in Gaza Strip, Palestine. Methods: This cross sectional study was conducted among a representative sample of Palestinian hypertensive patients (both genders, aged 30 - 64 years), patients receiving care in the primary healthcare centers in Gaza Strip, Palestine. Dietary patterns were obtained using factor analysis. Data regarding other variables was collected using an interview-based questionnaire. Statistical analysis was performed using SPSS version 20. Results: Two major dietary patterns were identified, including: Asian-like pattern and sweet-soft drinks-snacks pattern. After adjustment of potential confounders, patients in the lowest tertile of the Asian-like pattern had a lower odds for high systolic blood pressure, (OR 0.970 CI 95\% (.951-.990)), (P value < 0.05). No significant association was found between the Asian-like pattern with diastolic blood pressure. In addition, no significant associations were found between the sweet-soft drinks snacks pattern and blood pressure. Conclusion: The Asian-like pattern characterized by a high consumption of whole grains, potatoes, vegetables, fruit and olive may be associated with a lower prevalence of systolic blood pressure among hypertensive patients. Further future studies are required to confirm these findings.
\end{abstract}

Keywords: Blood Pressure, Dietary Patterns, Factor Analysis, Hypertension, Palestine

\section{Introduction}

Silent killer, hypertension or raised blood pressure (BP) is an important global health challenge [1-3]. Hypertension is the leading preventable risk factor for premature death and disability worldwide [3]. Raised BP is a major risk factor for coronary heart disease and ischemic as well as hemorrhagic stroke [4]. In addition, complications of raised BP include heart failure, peripheral vascular disease, renal impairment, retinal hemorrhage and visual impairment [4]. Globally, cardiovascular disease accounts for approximately 17 million deaths a year, nearly one third of the total [5]. Hypertension account for 9.4 million deaths worldwide every year [6]. Hypertension is responsible for at least $45 \%$ of deaths due to heart disease, and $51 \%$ of deaths due to stroke [5]. Globally, the World Health Organization estimates that, the overall prevalence of hypertension was around $40 \%$ in adults [4]. It disproportionately affects populations in low- and middleincome countries where health systems are weak [7]. In Palestine, the prevalence of hypertension was $27.6 \%$ among the registered Palestinian refugees [8]. Treating systolic and diastolic BP until they are less than $140 / 90 \mathrm{mmHg}$ is associated with a reduction in cardiovascular complications [4]. Addressing behavioral risk factors, e.g. unhealthy diet, 
harmful use of alcohol and physical inactivity, can prevent hypertension [7]. Recently, diet-disease relations have been recommended to be looked for through dietary pattern approach [9-11]. Dietary pattern analysis allows consideration of the entire diet, rather than individual foods or ingredients [12]. Furthermore, the dietary pattern approach reflects individuals dietary behaviors and therefore can provide more detailed information about nutritional etiology of chronic diseases [9-11]. Diet is one of the lifestyle factors that may play an essential role in preventing and managing of hypertension [13-14]. Furthermore, few studies have examined the association between dietary patterns and BP control. Most of the previous studies have examined the associations between a single food or nutrients and hypertension [15-18]. Therefore, understanding the relationship between dietary patterns with BP control may be useful in decreasing hypertension-related morbidity and mortality among hypertensive patients. To the best of our knowledge, this is the first study, which examined this association among hypertensive patients in Gaza Strip, Palestine. The purpose of the current study was to identify major dietary patterns among hypertensive patients and its association with BP control.

\section{Methods}

\subsection{Study Population}

The study population comprised of 765 hypertensive patients, aged 30 - 64 years, were included in a cross sectional study. In the years 2015 and 2016, a representative sample of participants were selected by a cluster random sampling methods from the primary healthcare centers (PHCs) in Gaza Strip, Palestine. The total number of PHCs in Gaza Strip is fifty-four [19]. Gaza Strip is divided into five smaller governorates and the study sample in our study was divided according to the number of PHCs in each governorate. Pregnant, nursing mothers and participants with other type of serious diseases like cancer, acute myocardial infarction or end-stage kidney disease were eliminated from the study. The study protocol was approved by the Ethics Committee of Tehran University of Medical Sciences and by the Palestinian Health Research Council. Furthermore, a written informed consent was obtained from all participants. Confidentiality was respected throughout the study.

\subsection{Assessment of Anthropometric Measurements}

Height $(\mathrm{cm})$, weight $(\mathrm{kg})$ and waist circumference $(\mathrm{cm})$, were measured for all participants using standard methods [20]. In addition, body mass index (BMI) was estimated by dividing weight $(\mathrm{kg})$ by the square of height in meters.

\subsection{Blood Tests and Lab Analysis}

After 12 hours fasting, blood sample of all participants were collected through venipuncture by a registered nurses and was used for blood chemistry analysis. Serum was separated immediately, and the extracted serum was investigated for (High-Density Lipoprotein Cholesterol (HDL-c) mg/dl and Triglycerides (TGs) mg/dl). The laboratory tests were analyzed in a licensed laboratory.

\subsection{Blood Pressure Assessment}

The BP measurement was took from the left arm (mmHg) using a stethoscope and mercury sphygmomanometer. Three readings on different days, while the patient was seated after relaxing for at least fifteen minutes in a quiet environment, empty bladder, without having smoked or consumed any coffee, tea, beverage that contains high levels of caffeine or any other food. The average of three measurements was recorded. In the present study, raised BP (Hypertension), was defined according to the World Health Organization definition, as a systolic BP equal to or above $140 \mathrm{mmHg}$ and/or diastolic BP equal to or above $90 \mathrm{mmHg}$ or treatment of previously diagnosed hypertension [4].

\subsection{Dietary Assessment}

A comprehensive data regarding dietary patterns were collected by an expert nutritionist, using a validated semiquantitative food frequency questionnaire (FFQ). The FFQ is relatively easy and inexpensive to administer and can be used to measure dietary intake over a prolonged time period [21-22]. In the present study the FFQ contains a list of 98 food items; it was developed and validated among Palestinian population in 2014 [23]. In our study, the method of dietary patterns assessment was published in the previous study [20]. Furthermore, the major dietary patterns were obtained using factor analysis after the classification of food items into 25 groups (Table 1).

\subsection{Covariates Assessment}

The socioeconomic, demographic and behavioral data were collected through an interview-based questionnaire. Past medical history and the usage of antihypertensive medications was also investigated. In addition, reports and all relevant documentation, including medical records were checked. Data about physical activity were collected using the International Physical Activity Questionnaire (IPAQ short version) [24]. The internationally accepted protocol was used to estimate the weekly calorie expenditure expressed as metabolic equivalents per week (MET/wk) [24]. According to the IPAQ scoring protocol, the patients were classified based on their weekly energy expenditure as follows: Insufficiently Active (IA) $\leq 600 \mathrm{MET} / \mathrm{wk}$; Sufficiently Active (SA) 601 to $1500 \mathrm{MET} / \mathrm{wk}$, and Very Active (VA) $\geq 1500$ MET/wk [25]. Pilot study was conducted on thirty patients to evaluate the tools of the study. Then, the tools of the study were modified according to the result of the pilot study.

\subsection{Statistical Analysis}

All statistical analysis was performed using SPSS version 20. Dietary intakes were converted into grams per day. Factor analysis was performed to determine the major dietary patterns among hypertensive patients. Factor analysis is a useful multivariable statistical tool for investigating dietary patterns 
[26-27]. It allows researchers to investigate concepts that are not easily measured directly by collapsing a large number of variables into a few interpretable underlying factors [28-29]. This data reduction method identifies independent vectors of variables in a correlation matrix and provides scores that allow individuals to be ranked in terms of how closely they conform to the total pattern [30]. In our study, the 98 food items in the FFQ were classified into 25 food groups (Table 1). The food grouping was based on the similarity of nutrient profiles and was somewhat similar to that used in previous studies [31-32]. A varimax rotation was used to determine the dietary patterns. For defining food groups in each pattern and simplifying dietary pattern tables, factor loads under 0.2 were excluded [33]. The factor load shows the association between food groups and dietary patterns. For determining the number of factors, we considered eigenvalues $>1$, the scree plot, and the interpretability of the factors. When a food group was loaded in more than one dietary pattern, only the pattern with a higher factor load was considered in the analysis. A factor score for the two major dietary patterns was calculated. This score for each individual shows, the extent to which the dietary pattern is consistent with one of the specified patterns. Higher factor scores show greater consumption of food groups in the pattern and vice versa. The adequacy of data was evaluated based on the value of Kaiser-Meyer-Olkin and Bartlett's test. The Kaiser-Mayer-Olkin coefficient, which represents the adequacy of the sample size for factor analysis and should be greater than 0.5 , was calculated and the obtained value was 0.743 in our study. The obtained dietary patterns scores are expressed as tertiles. The chi-square test was used to determine the significant differences between different categorical variable. The differences between mean were tested by independent samples t-test and one-way ANOVA. Moreover, the odds ratio (OR) and confidence interval (CI) for systolic and diastolic BP across tertiles categories of dietary pattern scores were tested by binary logistic regression. $\mathrm{P}$ value less than 0.05 was considered as statistically significant.

\section{Results}

A total of 765 patients with hypertension aged 30 to 64 years old $(62.2 \%$ females, $37.8 \%$ males $)$ were included in this study. The characteristics of the study population by sex are shown in Table 2. The results revealed that the mean age (years) for male patients was $54.78 \pm 7.2$ vs. $55.49 \pm 6.9$ for females. In addition, for the following factors (Educational level, employment history, family size, monthly income, history of smoking, physical activity level (Total MET), BMI $\left(\mathrm{kg} / \mathrm{m}^{2}\right)$, lipid abnormality or take medications for lipid abnormality, and HDL-c level), the difference was statistically significant in both sexes ( $\mathrm{P}$ value $<0.05$ for all). Then, the food consumption data for the 25 food groups (Table 1) were entered into the SPSS for factor analysis.

Table 1. Food Groupings used in the Dietary Pattern Analysis.

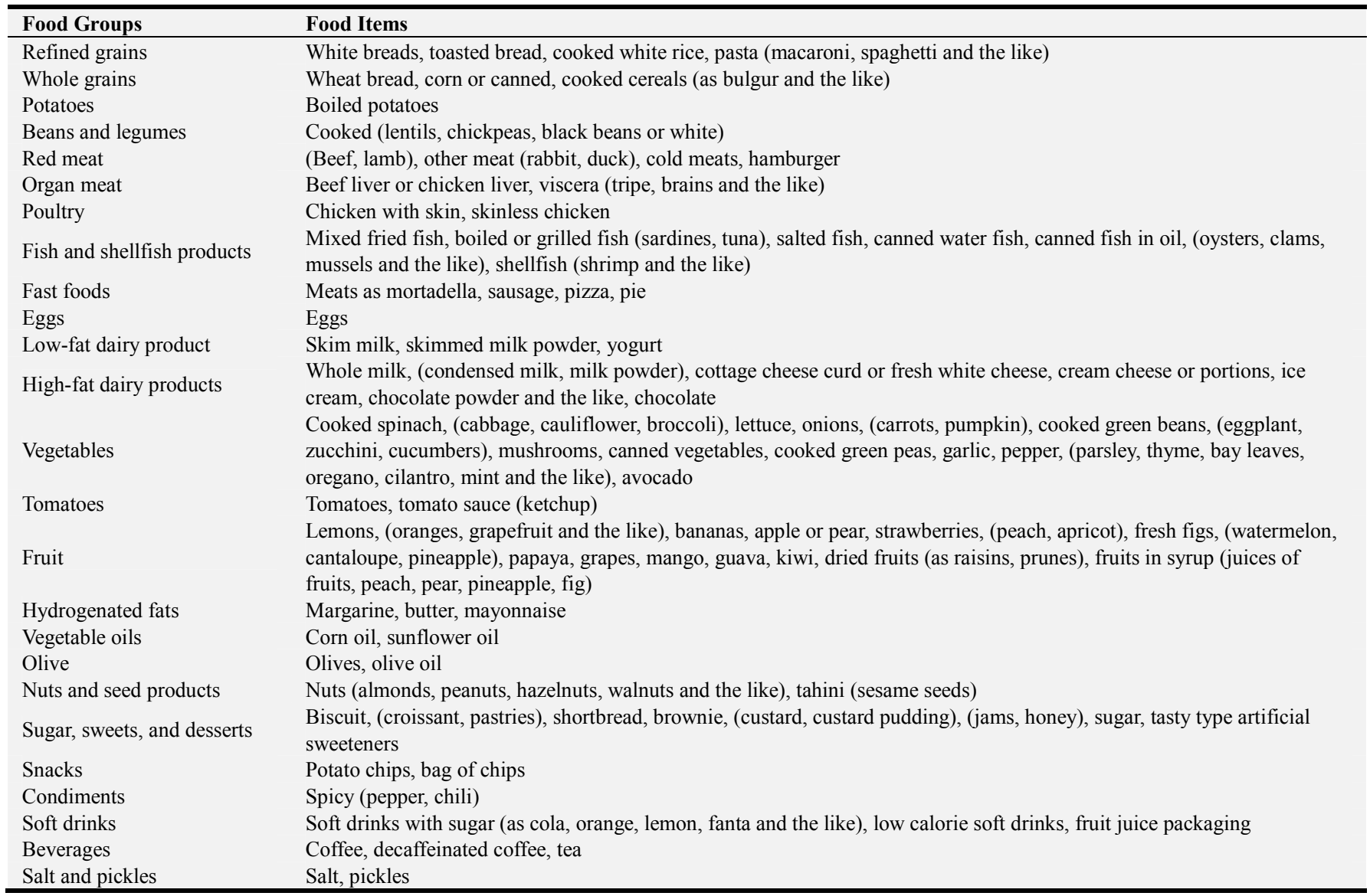


Table 2. Characteristics of the study population by sex.

\begin{tabular}{|c|c|c|c|c|c|}
\hline \multirow[t]{2}{*}{ Variables } & & \multirow{2}{*}{$(n=765)$} & \multirow{2}{*}{$(n=289)$} & \multirow{2}{*}{$\begin{array}{l}(n=476) \\
\text { No. }(\%)\end{array}$} & \multirow[t]{2}{*}{ P Value } \\
\hline & & & & & \\
\hline Age (years) & Mean \pm SD & $55.22 \pm 7.0$ & $54.78 \pm 7.2$ & $55.49 \pm 6.9$ & 0.604 \\
\hline \multirow{2}{*}{ Marital status } & Married & $754(98.6)$ & $286(37.9)$ & $468(62.1)$ & \multirow{2}{*}{0.350} \\
\hline & Unmarried & $11(1.4)$ & $3.0(27.3)$ & $8.0(72.3)$ & \\
\hline \multirow{2}{*}{ Educational level } & Low education & $451(59.0)$ & $140(31.0)$ & $311(69)$ & \multirow{2}{*}{0.001} \\
\hline & High education & $314(41.0)$ & $149(47.5)$ & $165(52.5)$ & \\
\hline \multirow{2}{*}{ Employment history } & Yes & $82(10.7)$ & $62(75.6)$ & $20(24.4)$ & \multirow{2}{*}{0.001} \\
\hline & No & $683(89.3)$ & $227(33.3)$ & $456(66.7)$ & \\
\hline \multirow{2}{*}{ Family size } & Less than five & $218(28.5)$ & $66(30.3)$ & $152(69.7)$ & \multirow{2}{*}{0.004} \\
\hline & Five or more & $547(71.5)$ & $223(40.8)$ & $324(59.2)$ & \\
\hline \multirow{2}{*}{ Monthly income } & $\leq 2000$ (NIS) & $711(92.9)$ & $251(35.3)$ & $460(64.7)$ & \multirow{2}{*}{0.001} \\
\hline & $>2000$ (NIS) & $54(7.1)$ & $38(70.4)$ & $16(29.6)$ & \\
\hline \multirow{2}{*}{ History of smoking } & Yes & $97(12.7)$ & $96(99.0)$ & $1.0(1.0)$ & \multirow{2}{*}{0.001} \\
\hline & No & $668(87.3)$ & $193(28.9)$ & $475(71.1)$ & \\
\hline History of alcohol intake & No & $765(100.0)$ & $289(37.8)$ & $476(62.2)$ & - \\
\hline Physical activity (Total MET) & Mean \pm SD & $808.1 \pm 1046$ & $1005.0 \pm 1098$ & $688.59 \pm 994.9$ & 0.019 \\
\hline Insufficiently Active (MET/wk) & $(\leq 600)$ & $445(58.2)$ & $133(29.9)$ & $312(70.1)$ & 0.001 \\
\hline Sufficiently Active (MET/wk) & (601 to 1500$)$ & $214(28.0)$ & $102(47.7)$ & $112(52.3)$ & 0.001 \\
\hline Very Active (MET/wk) & $(\geq 1500)$ & $106(13.9)$ & $54(50.9)$ & $52(49.1)$ & 0.002 \\
\hline Body mass index $\left(\mathrm{kg} / \mathrm{m}^{2}\right)$ & Mean \pm SD & $32.83 \pm 5.8$ & $30.3 \pm 4.8$ & $34.3 \pm 5.8$ & 0.011 \\
\hline Normal weight (BMI: $\mathrm{kg} / \mathrm{m}^{2}$ ) & (18.5 to 24.9$)$ & $54(7.1)$ & $40(74.1)$ & $14(25.9)$ & \multirow{3}{*}{0.001} \\
\hline Overweight (BMI: kg/m²) & (25 to 30$)$ & $196(25.6)$ & $105(53.6)$ & $91(46.4)$ & \\
\hline Obesity (BMI: kg/m²) & $(>30)$ & $515(67.3)$ & $144(28.0)$ & $371(72.0)$ & \\
\hline Waist circumference $(\mathrm{cm})$ & Mean \pm SD & $112.7 \pm 13.6$ & $107.9 \pm 12.8$ & $115.6 \pm 13.3$ & 0.157 \\
\hline \multirow{2}{*}{$\begin{array}{l}\text { Lipid abnormality or take medications for lipid } \\
\text { abnormality }\end{array}$} & Yes & $482(63.0)$ & $155(32.2)$ & $327(67.8)$ & \multirow{2}{*}{0.001} \\
\hline & No & $283(37.0)$ & $134(47.3)$ & $149(52.7)$ & \\
\hline Triglycerides level (mg/dl) & Mean \pm SD & $180.8 \pm 54.8$ & $173.2 \pm 55.2$ & $185.3 \pm 54.1$ & 0.405 \\
\hline HDL-cholesterol level (mg/dl) & Mean \pm SD & $41.86 \pm 7.5$ & $39.9 \pm 6.3$ & $43.0 \pm 8.0$ & 0.001 \\
\hline Systolic BP $(\mathrm{mmHg})$ & Mean \pm SD & $139.8 \pm 7.5$ & $138.9 \pm 7.1$ & $140.4 \pm 7.7$ & 0.297 \\
\hline Diastolic BP (mmHg) & Mean \pm SD & $88.27 \pm 5.5$ & $87.5 \pm 5.2$ & $88.7 \pm 5.6$ & 0.051 \\
\hline
\end{tabular}

Data are expressed as means $\pm \mathrm{SD}$ for continuous variables and as percentage for categorical variables. The differences between means were tested by using independent sample $t$ test. The chi-square test was used to examine differences in the prevalence of different categorical variable. $\mathrm{P}$ value less than 0.05 was considered as statistically significant. SD, stander deviation.

The scree plot of eigenvalues indicated two major patterns:
1) Asian-like pattern characterized by a high intake of whole grains, potatoes, vegetables, fruit and olive as well as a low intake of refined grains, sugar, sweets and desserts; 2) Sweetsoft drinks-snacks pattern characterized by a high intake of refined grains, sugar, sweets, desserts, snacks, soft drinks and beverage as well as a low intake of fruits. The factor loading matrixes for the two major patterns are shown in Table 3.

Table 3. Factor loading matrix for major dietary patterns.

\begin{tabular}{lll}
\hline \multirow{2}{*}{ Food Groups } & Dietary patterns & Asian-like pattern \\
\cline { 2 - 3 } & Sweet-soft drinks-snacks pattern & 0.238 \\
\hline Refined grains & 0.258 & 0.251 \\
Whole grains & - & 0.213 \\
Potatoes & - & - \\
Beans and legumes & - & - \\
Red meat & - & - \\
Organ meat & - & - \\
Poultry & - & - \\
Fish and shellfish products & - & - \\
Fast foods & - & - \\
Eggs & - & - \\
Low-fat dairy product & - & - \\
High-fat dairy products & - & 0.287 \\
Vegetables & - & - \\
Tomatoes & - & 0.965 \\
Fruit & 0.261 & - \\
Hydrogenated fats & - & - \\
Vegetable oils & - & 0.208 \\
Olive & - & - \\
Nuts and seed products & - & \\
\hline
\end{tabular}




\begin{tabular}{lll}
\hline \multirow{2}{*}{ Food Groups } & Dietary patterns & \\
\cline { 2 - 3 } & Sweet-soft drinks-snacks pattern & Asian-like pattern \\
\hline Sugar, sweets, and desserts & 0.307 & 0.246 \\
Snacks & 0.245 & - \\
Condiments & - & - \\
Soft drinks & 0.999 & - \\
Beverages & 0.236 & - \\
Salt and pickles & - & - \\
Variance explained (\%) & 26.024 & 60.021 \\
Values less than 0.2 were omitted for simplicity. Total variance explained by two factors: 86.045 & \\
\hline
\end{tabular}

These two major dietary patterns explained $60.0 \%$ and $26.0 \%$ of the total variance, respectively. In our study, the dietary patterns scores were classified as tertiles. Then, the characteristics of the study population were evaluated within the tertiles. Table 4 shows that patients in the lowest tertile (T1) of the Asian-like pattern were had a small family size (less than five) (31.7 vs. $34.4 \%$, P value $<0.005)$, had a lower BMI $\left(32.6 \pm 5.9\right.$ vs. $33.1 \pm 5.5 \mathrm{~kg} / \mathrm{m}^{2}, \mathrm{P}$ value $\left.<0.05\right)$, and had a lower lipid abnormality or take medications for lipid abnormality (33.8 vs. $34.7 \%$, P value $<0.05)$, compared to those in the highest tertile (T3). On the other hand, only the distribution of patients with regard to history of smoking and physical activity level (Total MET) was significantly different across the tertiles of the sweet-soft drinks-snacks pattern ( $\mathrm{P}$ value $<0.05)$.

Table 4. Characteristics and dietary intakes of study population by Tertile (T) categories of dietary pattern scores.

\begin{tabular}{|c|c|c|c|c|c|c|c|c|}
\hline \multirow{2}{*}{ Variables } & \multicolumn{3}{|c|}{ Sweet-soft drinks-snacks pattern } & \multirow{2}{*}{ P Value } & \multicolumn{3}{|c|}{ Asian-like pattern } & \multirow{2}{*}{ P Value } \\
\hline & T1 & $\mathbf{T 2}$ & T3 & & T1 & $\mathbf{T 2}$ & T3 & \\
\hline \multicolumn{9}{|l|}{ Age (years) } \\
\hline Mean \pm SD & $55.0 \pm 7.1$ & $55.2 \pm 6.5$ & $55.2 \pm 7.4$ & 0.897 & $55.5 \pm 7.2$ & $55.6 \pm 6.8$ & $54.5 \pm 7.0$ & 0.051 \\
\hline \multicolumn{9}{|l|}{ Gender \% } \\
\hline Males & 32.1 & 32.9 & 35.0 & \multirow{2}{*}{0.585} & 36.3 & 29.1 & 34.6 & \multirow{2}{*}{0.855} \\
\hline Females & 34.0 & 33.6 & 32.4 & & 31.5 & 35.9 & 32.6 & \\
\hline \multicolumn{9}{|l|}{ Marital status \% } \\
\hline Married & 33.4 & 33.3 & 33.3 & \multirow{2}{*}{0.532} & 33.3 & 33.4 & 33.3 & \multirow{2}{*}{0.726} \\
\hline Unmarried & 27.2 & 36.4 & 36.4 & & 36.4 & 27.2 & 36.4 & \\
\hline \multicolumn{9}{|c|}{ Educational level \% } \\
\hline Low education & 36.6 & 30.4 & 33.0 & \multirow{2}{*}{0.923} & 32.1 & 33.7 & 34.2 & \multirow{2}{*}{0.133} \\
\hline High education & 28.7 & 37.6 & 33.7 & & 35.0 & 32.8 & 32.2 & \\
\hline \multicolumn{9}{|c|}{ Employment history \% } \\
\hline Yes & 32.9 & 37.8 & 29.3 & 0.560 & 36.6 & 23.2 & 40.2 & 0.636 \\
\hline \multicolumn{9}{|l|}{ Family size $\%$} \\
\hline Less than five & 32.1 & 33.0 & 34.9 & \multirow{2}{*}{0.062} & 31.7 & 33.9 & 34.4 & \multirow{2}{*}{0.005} \\
\hline Five or more & 33.9 & 33.4 & 32.7 & & 34.1 & 33.0 & 32.9 & \\
\hline \multicolumn{9}{|c|}{ Monthly income (NIS) \% } \\
\hline$\leq 2000$ (NIS) & 32.9 & 33.5 & 33.6 & \multirow{2}{*}{0.769} & 34.0 & 33.8 & 32.2 & \multirow{2}{*}{0.916} \\
\hline$>2000$ (NIS) & 38.9 & 31.5 & 29.6 & & 24.0 & 27.8 & 48.2 & \\
\hline \multicolumn{9}{|c|}{ History of smoking $\%$} \\
\hline Yes & 36.0 & 28.9 & 35.1 & 0.039 & 34.0 & 30.9 & 35.1 & 0.545 \\
\hline \multicolumn{9}{|c|}{ Physical activity (Total MET) } \\
\hline Mean \pm SD & $815 \pm 1066$ & $737 \pm 1046$ & $871 \pm 1024$ & 0.001 & $881 \pm 1224$ & $819 \pm 922$ & $723 \pm 962$ & 0.195 \\
\hline Insufficiently Ac & $600 \mathrm{MET} / \mathrm{wl}$ & & & & & & & \\
\hline Yes & 33.0 & 34.8 & 32.2 & 0.857 & 31.9 & 33.5 & 34.6 & 0.210 \\
\hline Sufficiently Acti & to $1500 \mathrm{ME}$ & & & & & & & \\
\hline Yes & 34.1 & 32.7 & 33.2 & 0.731 & 36.0 & 30.8 & 33.2 & 0.310 \\
\hline Very Active $(\geq 1$ & $\mathrm{T} / \mathrm{wk})$ & & & & & & & \\
\hline Yes & 33.0 & 28.3 & 38.7 & 0.849 & 34.0 & 37.7 & 28.3 & 0.638 \\
\hline Body mass index & & & & & & & & \\
\hline Mean \pm SD & $33.6 \pm 6.0$ & $32.3 \pm 5.2$ & $32.4 \pm 6.0$ & 0.591 & $32.6 \pm 5.9$ & $32.6 \pm 5.9$ & $33.1 \pm 5.5$ & 0.044 \\
\hline Waist circumfere & & & & & & & & \\
\hline Mean \pm SD & $112.8 \pm 13$ & $113.5 \pm 13$ & $111.8 \pm 13$ & 0.409 & $113.2 \pm 13$ & $112.6 \pm 14$ & $112.4 \pm 12$ & 0.731 \\
\hline Lipid abnormalit & e medicatior & ipid abnorm & & & & & & \\
\hline Yes & 33.4 & 35.0 & 31.5 & 0.200 & 33.8 & 31.5 & 34.7 & 0.021 \\
\hline Triglycerides lev & & & & & & & & \\
\hline Mean \pm SD & $175.5 \pm 48$ & $180.8 \pm 50$ & $185.9 \pm 63$ & 0.966 & $187.7 \pm 62$ & $179.2 \pm 53$ & $175.4 \pm 46$ & 0.697 \\
\hline HDL-cholesterol & $\mathrm{ng} / \mathrm{dl})$ & & & & & & & \\
\hline Mean \pm SD & $41.7 \pm 7.2$ & $41.3 \pm 7.5$ & $42.4 \pm 7.9$ & 0.235 & $41.7 \pm 7.7$ & $43.3 \pm 7.7$ & $40.4 \pm 6.9$ & 0.177 \\
\hline
\end{tabular}

ANOVA test was used for quantitative variables and chisquare for qualitative variables. $\mathrm{P}$ value less than 0.05 was considered as statistically significant. SD, stander deviation.

Finally, the OR and CI for systolic and diastolic blood pressure across tertiles categories of dietary patterns scores were computed (Table 5). Our findings demonstrate that after adjustment for confounding variables, patients in the lowest tertile (T1) of the Asian-like pattern characterized by a high 
intake of whole grains, potatoes, vegetables, fruit and olive had a lower odds for high systolic BP, (OR 0.970 CI 95\% (.951.990)), (P value $<0.05)$. No significant association was found between the Asian-like pattern with diastolic BP. In addition, no significant association was found between the sweet-soft drinks snacks pattern with systolic and diastolic BP.

Table 5. Odd ratio and confidence interval for systolic and diastolic BP across tertiles categories of dietary pattern scores.

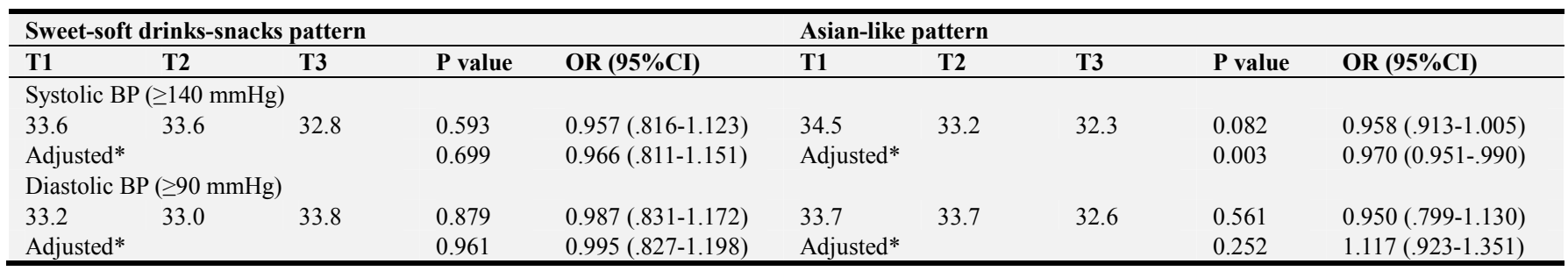

The OR and CI for systolic BP $(\geq 140 \mathrm{mmHg})$, and diastolic BP $(\geq 90 \mathrm{mmHg})$ across tertiles categories of dietary pattern scores were tested by binary logistic regression. *Adjusted for age, gender, marital status, educational level, employment history, family size, monthly income, history of smoking, physical activity (Total MET), BMI $\left(\mathrm{kg} / \mathrm{m}^{2}\right), \mathrm{WC}$ (cm), lipid abnormality or take medications for lipid abnormality, TGs level (mg/dl) and HDL-c level (mg/dl). P value less than 0.05 was considered as statistically significant. OR, odds ratio; CI, confidence interval.

\section{Discussion}

Cardiovascular diseases are the main leading cause of death among Palestinian adults, with high BP being a major determining factor [4-8]. Our study was conducted to determine the associations between major dietary patterns and BP control among hypertensive patients in Gaza Strip, Palestine. In this study, all participants had high BP $(\geq 140 / 90$ $\mathrm{mmHg}$ ) or treatment of previously diagnosed hypertension. To our knowledge, this is the first investigation reporting such an association among hypertensive patients in Gaza Strip, Palestine. In our study, two major dietary patterns were identified by factor analysis. 1) Asian-like pattern characterized by a high intake of whole grains, potatoes, vegetables, fruit and olive as well as a low intake of refined grains, sugar, sweets and desserts; 2) Sweet-soft drinks-snacks pattern characterized by a high intake of refined grains, sugar, sweets, desserts, snacks, soft drinks and beverage as well as a low intake of fruits. Our study findings showed that after adjustment for confounding variables, the Asian-like pattern may be associated with a lower prevalence of systolic BP among hypertensive patients. The dietary pattern approach reflects individuals dietary behaviors and therefore can provide more detailed information about nutritional etiology of chronic diseases [9-11]. Ndanuko et al. [34] in a meta-analysis identified three dietary patterns: Healthy dietary patterns such as the Dietary Approaches to Stop Hypertension (DASH) diet, Nordic diet and Mediterranean diet. These dietary patterns are rich in fruit, vegetables, whole grains, legumes, seeds, nuts, fish and dairy. The author concluded that these dietary patterns were inversely associated with high BP. The results of our study support these findings. The DASH dietary pattern is rich in fruits and vegetables, low fat dairy products, whole grains, fish, poultry, beans, seeds and nuts. It is low in sodium, added sugars, sweets, fats and red meats. The DASH dietary pattern is a recognized treatment for hypertension, stroke and heart disease [35]. Nilofer et al. [36] identified three dietary patterns among Pakistani low-income urban adults. The author concluded that the seafood and yogurt pattern (Characterized by fish, prawns, potatoes and yogurt) was less likely to be associated with hypertension, whereas no significant associations were seen for other two dietary patterns. Dong et al. [37] identified three major dietary patterns: Western, traditional northern and traditional southern. The author concluded that the traditional southern pattern (Characterized by high intakes of fruit, pork, poultry, rice, vegetables, aquatic products and nuts) was associated with lower prevalence of hypertension among Chinese adults. Ji-Ye Shin et al. [38] identified three major dietary patterns: traditional, western, and dairy and carbohydrate patterns. The author concluded that the dairy and carbohydrate pattern may be associated with a reduced risk of hypertension whereas the western pattern may be associated with an increased risk of hypertension among Korean adults. The previous dietary patterns are different from those obtained in our study. This can be explained by demographic, cultural and ethnic differences.

On the other hand, our findings show that patients in the lowest tertile (T1) of the Asian-like pattern were had a small family size (less than five), had a lower BMI, and had a lower lipid abnormality or take medications for lipid abnormality, compared to those in the highest tertile (T3). According to the DASH, diets high in fruits, vegetables and low in fat may be associated with a lower risk of hypertension, decreased weight, BMI, serum triglycerides, and very low density lipoprotein cholesterol levels [39-40]. The results of our study support these findings. In our study, the inverse association between Asian-like pattern with high systolic BP could be attributed to pattern's healthy ingredients including vitamins, dietary fibers, potassium, magnesium and antioxidants. These nutrients have been independently associated with reduced risks of high BP [41]. In our study, the Asian-like pattern has been shown to be the healthiest dietary pattern and is quite close to that diet, which generally recommended as a healthy dietary pattern with low intake of sodium, animal foods, saturated fat, trans fat, cholesterol and simple sugar, which may be associated with a higher risks of hypertension and its complications [42]. On 
contrary, no significant association was found between the Asian-like pattern with diastolic BP. Our study not adjusted for other confounding variables such as genetics factors, types of antihypertensive drugs and psychological factors, which could contribute to these results. The main limitations of this study is its cross sectional design; the causal relationship could not be determined. Moreover, the possibility of recall bias and misreporting by using FFQ assessment of dietary patterns are other limitations. Furthermore, regrettably we do not have tests of total cholesterol, and low-density lipoprotein cholesterol as a marker of BP control. The main strength of our study was its being the first study, which identified the major dietary patterns among hypertensive patients in Gaza Strip, Palestine, and its large sample size.

\section{Conclusions}

In conclusion, the Asian-like pattern characterized by a high consumption of whole grains, potatoes, vegetables, fruit and olive may be associated with a lower prevalence of systolic blood pressure among hypertensive patients. Further future studies are required to confirm these findings.

\section{Acknowledgements}

The authors wish to thank and appreciate the staff and participants in the Palestinian Ministry of Health, PHCs for their important participation in the study.

\section{References}

[1] Kearney PM, Whelton M, Reynolds K, Muntner P, Whelton PK, He J. Global burden of hypertension: analysis of worldwide data. The lancet. 2005;365(9455):217-23.

[2] Organization WH. Chronic Disease and Health Promotion. Global Infobase. Available at:

https://apps.who.int/infobase/Index.aspx. Accessed 1 December 2017.

[3] O'brien E, Pickering T, Asmar R, Myers M, Parati G, Staessen J, Mengden T, Imai Y, Waeber B, Palatini P, et al. Working Group on Blood Pressure Monitoring of the European Society of Hypertension International Protocol for validation of blood pressure measuring devices in adults. Blood pressure monitoring. 2002;7(1):3-17.

[4] Organization WH. Raised blood pressure. Global Health Observatory data. Available at: http://www.who.int/gho/ncd/risk factors/bloodpressure prevalencetext/en/. Accessed 1 December 2017.

[5] Organization WH. Causes of Death 2008. Available at: http ://www.who.int/healthinfo/global_burden_disease/cod_20 08_sources_methods.pdf. Accessed 1 December 2017.

[6] Lim SS, Vos T, Flaxman AD, Danaei G, Shibuya K, AdairRohani H, Amann M, Anderson HR, Andrews KG, Aryee M, et al. A comparative risk assessment of burden of disease and injury attributable to 67 risk factors and risk factor clusters in
21 regions, 1990-2010: a systematic analysis for the Global Burden of Disease Study 2010. The lancet. 2013;380(9859):2224-60.

[7] Organization WH. A Global Brief on Hypertension: Silent Killer. Global Public Health Crisis, World Health Organization (WHO), Geneva. 2013.

[8] Khdour M, Hallak H, Shaeen M, Jarab A, Al-Shahed Q. Prevalence, awareness, treatment and control of hypertension in the Palestinian population. Journal of human hypertension. 2013;27(10):623-8.

[9] Hu FB. Dietary pattern analysis: a new direction in nutritional epidemiology. Current opinion in lipidology. 2002;13(1):3-9.

[10] Kant AK. Dietary patterns and health outcomes. Journal of the American Dietetic Association. 2004;104(4):615-35.

[11] Newby P, Tucker KL. Empirically derived eating patterns using factor or cluster analysis: a review. Nutrition reviews. 2004;62(5):177-203.

[12] Council NR. Diet and health: implications for reducing chronic disease risk: National Academies Press; 1989.

[13] Conlin PR, Chow D, Miller ER, Svetkey LP, Lin P-H, Harsha DW, Moore TJ, Sacks FM, Appel LJ. The effect of dietary patterns on blood pressure control in hypertensive patients: results from the Dietary Approaches to Stop Hypertension (DASH) trial. American journal of hypertension. 2000;13(9):949-55.

[14] NIH U. Department of Health and Human Services, National Institutes of Health, National Heart, Lung, and Blood Institute, Your guide to lowering your blood pressure with DASH. DASH eating plan. 2006.

[15] Streppel MT, Arends LR, van't Veer P, Grobbee DE, Geleijnse JM. Dietary fiber and blood pressure: a meta-analysis of randomized placebo-controlled trials. Archives of internal medicine. 2005;165(2):150-6.

[16] Ha SK. Dietary salt intake and hypertension. Electrolytes \& Blood Pressure. 2014;12(1):7-18.

[17] Xiong X, Wang P, Li X, Zhang Y, Li S. The effects of red yeast rice dietary supplement on blood pressure, lipid profile, and C-reactive protein in hypertension: A systematic review. Critical reviews in food science and nutrition. 2017;57(9):1831-51.

[18] Borgi L, Muraki I, Satija A, Willett WC, Rimm EB, Forman JP. Fruit and vegetable consumption and the incidence of hypertension in three prospective cohort studies. Hypertension. 2015: HYPERTENSIONAHA. 115.06497.

[19] Ministry of health. The annual report of the hospital general administration. 2013; available at: http://www.moh.ps/?Lang=0\&page=1\&id=155. Accessed 1 May 2017.

[20] El Bilbeisi AH, Hosseini S, Djafarian K. Association of dietary patterns with diabetes complications among type 2 diabetes patients in Gaza Strip, Palestine: a cross sectional study. Journal of Health, Population and Nutrition. 2017; 36(1):37.

[21] Cade J, Thompson R, Burley V, Warm D. Development, validation and utilisation of food-frequency questionnaires-a review. Public health nutrition. 2002;5(4):567-87. 
[22] Wakai K. A review of food frequency questionnaires developed and validated in Japan. Journal of epidemiology. 2009;19(1):1-11.

[23] Hamdan M, Monteagudo C, Lorenzo-Tovar M-L, Tur J-A, Olea-Serrano F, Mariscal-Arcas M. Development and validation of a nutritional questionnaire for the Palestine population. Public health nutrition. 2014;17(11):2512-8.

[24] Craig CL, Marshall AL, Sjorstrom M, Bauman AE, Booth ML, Ainsworth BE, Pratt M, Ekelund U, Yngve A, Sallis JF, Oja P. International physical activity questionnaire: 12-country reliability and validity. Medicine and science in sports and exercise. 2003;35(8):1381-95.

[25] Ghasemi A, Tohidi M, Derakhshan A, Hasheminia M, Azizi F, Hadaegh F. Cut-off points of homeostasis model assessment of insulin resistance, beta-cell function, and fasting serum insulin to identify future type 2 diabetes: Tehran Lipid and Glucose Study. Acta diabetologica. 2015;52(5):905-15.

[26] Nicklas T, Webber L, Thompson B, Berenson G. A multivariate model for assessing eating patterns and their relationship to cardiovascular risk factors: the Bogalusa Heart Study. The American journal of clinical nutrition. 1989;49(6):1320-7.

[27] Hebert JR, Kabat GC. Implications for cancer epidemiology of differences in dietary intake associated with alcohol consumption. 1991.

[28] Kim J-O, Mueller CW. Factor analysis: Statistical methods and practical issues: Sage; 1978.

[29] Kleinbaum D, Kupper L, Nizam A, Rosenberg E. Applied regression analysis and other multivariable methods: Nelson Education; 2013.

[30] Jacques PF, Tucker KL. Are dietary patterns useful for understanding the role of diet in chronic disease?: Am Soc Nutrition; 2001.

[31] Abdollahi S, Zeinali F, Azam K, Toupchian O, Djafarian K. Identifying major dietary patterns among the elderly in Tehran health homes. Jundishapur Journal of Health Sciences. 2015;7(4).

[32] Hosseyni Esfahani F, Jazayeri A, Mirmiran P, Mehrabi Y, Azizi F. Dietary patterns and their association with sociodemographic and lifestyle factors among Thehrani adults: Tehran Lipid and Glucose Study. Journal of School of Public Health and Institute of Public Health Research. 2008;6(1):2336.
[33] ESMAEILLZADEH A, AZADBAKHT L, KHOSHFETRAT MR, KIMIAGAR M. Major dietary patterns, general and central adiposity among tehrani female teachers. 2011.

[34] Ndanuko RN, Tapsell LC, Charlton KE, Neale EP, Batterham MJ. Dietary patterns and blood pressure in adults: a systematic review and meta-analysis of randomized controlled trials. Advances in Nutrition: An International Review Journal. 2016;7(1):76-89.

[35] Sacks FM, Appel LJ, Moore TJ, Obarzanek E, Vollmer WM, Svetkey LP, Bray GA, Vogt TM, Cutler JA, Windhauser MM, et al. A dietary approach to prevent hypertension: a review of the Dietary Approaches to Stop Hypertension (DASH) Study. Clinical cardiology. 1999;22(S3):6-10.

[36] Safdar NF, Bertone-Johnson ER, Cordeiro L, Jafar TH, Cohen NL. Dietary patterns and their association with hypertension among Pakistani urban adults. Asia Pacific journal of clinical nutrition. 2015;24(4):710-9.

[37] Wang D, He Y, Li Y, Luan D, Yang X, Zhai F, and Guansheng Ma. Dietary patterns and hypertension among Chinese adults: a nationally representative cross-sectional study. BMC public health. 2011;11(1):925.

[38] Shin J-Y, Kim J-M, Kim Y. Associations between dietary patterns and hypertension among Korean adults: the Korean National Health and Nutrition Examination Survey (20082010). Nutrition research and practice. 2013;7(3):224-32.

[39] Appel LJ, Moore TJ, Obarzanek E, Vollmer WM, Svetkey LP, Sacks FM, Bray GA, Vogt TM, Cutler JA, Windhauser MM, et al. A clinical trial of the effects of dietary patterns on blood pressure. New England Journal of Medicine. 1997;336(16):1117-24.

[40] Asemi Z, Samimi M, Tabassi Z, Shakeri H, Sabihi S-S, Esmaillzadeh A. Effects of DASH diet on lipid profiles and biomarkers of oxidative stress in overweight and obese women with polycystic ovary syndrome: a randomized clinical trial. Nutrition. 2014;30(11):1287-93.

[41] Pischke CR, Weidner G, Elliott-Eller M, Scherwitz L, MerrittWorden TA, Marlin R, Lee Lipsenthal, Robert C. Finkel, Donald Saunders, Patty McCormac, et al. Comparison of coronary risk factors and quality of life in coronary artery disease patients with versus without diabetes mellitus. The American journal of cardiology. 2006;97(9):1267-73.

[42] Williams DE, Prevost AT, Whichelow MJ, Cox BD, Day NE, Wareham NJ. A cross-sectional study of dietary patterns with glucose intolerance and other features of the metabolic syndrome. British Journal of Nutrition. 2000;83(3):257-66. 